\title{
Band effect: A possible mechanism for large magnetoresistance in nonmagnetic materials
}

\author{
Gang Xiong \\ Department of Physics, Hong Kong University of Science and Technology, Hong Kong, China \\ and Department of Physics, Wuhan University, Wuhan 430072, China \\ Shi-Dong Wang and X. R. Wang \\ Department of Physics, Hong Kong University of Science and Technology, Hong Kong, China
}

(Received 13 December 1999)

\begin{abstract}
Motivated by a recently observed unusual magnetoresistance in sliver chalcogenides, we propose a mechanism for large magnetoresistance in nonmagnetic materials. We show that the Zeeman splitting can play a very important role in MR under certain conditions. In general, the electron density of states can be divided into two parts, one for spin-up electrons and the other for spindown. The two parts will shift against each other in an external magnetic field due to the Zeeman effect, leading to a substantial change in the effective carrier density when the original Fermi level is near the edge of the band and the Lande factor $g$ of electrons is large such that the electron density of states at the Fermi level is sensitive to the external magnetic field.
\end{abstract}

Recently, there has been great interest in studying large magnetoresistance $(\mathrm{MR})^{1-4}$ because of its potential applications in information industry. Both giant magnetoresistance $(\mathrm{GMR})^{1-3}$ and colossal magnetoresistance (CMR) (Ref. 4) are found in layered structures and granular systems containing magnetic elements. However, the reliability of magnetic materials related to the magnetic domains and the magnetic hysteresis limits its application. This limitation leads to a great deal of interest in nonmagnetic materials with large MR in recent years. Liu and Chien ${ }^{5}$ found a large positive MR in bismuth nanowires. Thio et al. ${ }^{6}$ reported a large positive $\mathrm{MR}$ in zero-band-gap $\mathrm{Hg}_{1-x} \mathrm{Cd}_{x} \mathrm{Te}$. $\mathrm{Xu}$ et al. ${ }^{7}$ discovered a large positive MR in nonmagnetic silver chalcogenides with slightly altered stoichiometry. The mechanism for the large MR in silver chalcogenides is still unknown while the large MR in bismuth nanowires can be explained by the Lorentz deflection of electrons with an excessively long elastic mean free time, ${ }^{5}$ and the large MR in zero-bandgap $\mathrm{Hg}_{1-x} \mathrm{Cd}_{x} \mathrm{Te}$ materials can be understood by a twocarrier model. 6

The recently observed large MR in silver chalcogenides has some strange features. ${ }^{7}$ The materials show no appreciable MR with ideal stoichiometry, $\mathrm{Ag}_{2} \mathrm{Se}$ and $\mathrm{Ag}_{2} \mathrm{Te}$, while large MR appears with only a small alteration of the stoichiometry, $\mathrm{Ag}_{2}+\delta$ Se and $\mathrm{Ag}_{2}+{ }_{\delta}$ Te with $\delta$ being as small as 0.01 . In fact, MR decreases when $\delta$ is further increased. At high field of tens of $\mathrm{kOe}, \mathrm{MR}$ is positive and reaches a quite high value of above $100 \%$ even at temperatures as high as $300 \mathrm{~K}$. It is known that $\mathrm{Ag}_{2} \mathrm{Se}$ and $\mathrm{Ag}_{2} \mathrm{Te}$ are intrinsic narrow-gap semiconductors while there should be a few electrons in the conduction band for $\mathrm{Ag}_{2}+\delta$ Se and $\mathrm{Ag}_{2}+\delta$ Te. For magnetic materials, GMR is associated with the spin-related scattering process. The picture is that spin-up and spin-down electrons are scattered by the magnetic components with different scattering rates. When the magnetic field is increased, magnetic moments in the system are gradually aligned such that one of the scattering rates decreases. Thus, resistance is reduced (negative MR) and GMR results. On the other hand, it is believed that CMR is related to the metal-insulator transition induced by the magnetic field though the detailed mechanism is still illusive.

Obviously, the mechanisms originated from magnetic spin cannot be applied to the silver chalcogenides. Neither does the mechanisms for bismuth nanowires and for zeroband-gap $\mathrm{Hg}_{1-x} \mathrm{Cd}_{x} \mathrm{Te}$ apply. Furthermore, the mechanism proposed by one of the authors ${ }^{8}$ for GMR in a granular material or a quantum dot array containing nonmagnetic elements cannot explain the large MR in silver chalcogenides, either. Therefore, there must be a new unknown mechanism for large magnetoresistance. In this paper, we propose a nonuniversal mechanism for a large MR. We show that a nonmagnetic material with a large Lande factor $g$ may have a very large positive MR when the electron density of states at the Fermi level is very sensitive to an external magnetic field. The possible candidates with such properties are semimetals and/or lightly doped narrow-gap semiconductors such as silver chalcogenides. It is known that band structure of a material depends on methods of the material preparation. Thus, this mechanism should also be sensitive to the details in material preparation. In this sense, the mechanism proposed here is not universial. It is interesting to notice that a recent experiment ${ }^{9}$ on the silver chalcogenides shows an MR substantially smaller than that of $\mathrm{Xu}$ et al. ${ }^{7}$ while another experiment on the silver chalcogenides prepared by vapor deposition method does not show any pronounced MR. ${ }^{10}$

According to the standard electronic transport theory of an electron gas, only the electrons near the Fermi level can participate in electron conduction. Therefore, the electron conductivity depends on the electron density of states (DOS) at the Fermi level, the electron scattering time $\tau$, and the electron effective mass $m$. In general, $\tau$ and $m$ are not very sensitive to an external magnetic field in nonmagnetic materials. One expects that the dominant source of large MR comes from the magnetic-field dependence of the electron DOS at the Fermi level. Thus, the conductivity $\sigma$ is

$$
\sigma \propto \rho\left(E_{F}\right)
$$




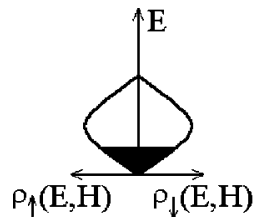

(a)

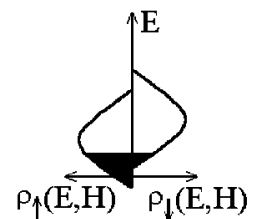

(b)

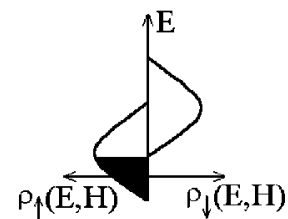

(c)
FIG. 1. Illustrations of the change of the Fermi level $E_{F}$ induced by the Zeeman splitting. The parts filled with black color are occupied by carriers. (a) $H=0$; (b) $0<H<H_{c}$; (c) $H>H_{c}$ when all electrons are in spin-up part. The electron energy $E$ is measured from the bottom of the band at zero field.

where $\rho\left(E_{F}\right)$ is the electron DOS at the Fermi level $E_{F}$. What we need to study is how an external magnetic field $H$ influences $\rho\left(E_{F}\right)$ so that a large MR may appear under certain conditions. In general, the electron DOS $\rho(E, H)$ can be divided into two parts, one for spin-up electrons, i.e., electron- spin component along the field $H$ being $S_{z}=1$, and the other for spin-down

$$
\rho(E, H)=\rho_{\uparrow}(E, H)+\rho_{\downarrow}(E, H),
$$

where $\rho_{\uparrow}(E, H)$ and $\rho_{\downarrow}(E, H)$ denote the DOS for spin-up and spin-down electrons, respectively. $\rho_{\uparrow}(E, H)$ and $\rho_{\downarrow}(E, H)$ are identical in the absence of a magnetic field because electronic states with up-spin and down-spin are degenerated. This is illustrated in Fig. 1(a). We shall define $\rho_{0}(E)$ as

$$
\rho_{\uparrow}(E, H=0)=\rho_{\downarrow}(E, H=0) \equiv \rho_{0}(E) .
$$

Thus, we have

$$
\rho(E, H=0)=2 \rho_{0}(E) .
$$

The energy is measured by choosing the bottom of the conduction band at zero field as zero, that is, $\rho_{0}(E)=0$ when $E$ is less than zero or larger than the width of the conduction band.

In an external magnetic field $H$, the Zeeman energy $\Delta \epsilon$ $=-g \mu_{B} H S_{z}$ is added into the electron energy, where $g$ is the Lande factor of electrons, and $\mu_{B}$ is the Bohr magneton. As the Zeeman energy $\Delta \epsilon$ is a constant for electrons with the same $S_{z}$, the shape of the DOS for spin-up and spin-down electrons remain the same. However, as shown in Fig. 1(b), $\rho_{\uparrow}(E, H)$ shifts downward by the Zeeman energy $|\Delta \epsilon|$ while $\rho_{\downarrow}(E, H)$ shifts upward by the Zeeman energy $|\Delta \epsilon|$ in comparison with those at zero field, that is, $\rho_{\uparrow}(E, H)=\rho_{0}(E$ $+|\Delta \epsilon|)$ and $\rho_{\downarrow}(E, H)=\rho_{0}(E-|\Delta \epsilon|)$. Thus, one obtains

$$
\rho(E, H)=\rho_{0}(E-|\Delta \epsilon|)+\rho_{0}(E+|\Delta \epsilon|) .
$$

Due to the splitting, some spin-down electrons will be converted into spin-up state. Thus, more electrons will be in the spin-up states. One expects that the Fermi level will also change with the Zeeman splitting which in turn depends on the strength of the magnetic field. At zero temperature, and considering the conservation of total electron number, one obtains

$$
\int_{0}^{E_{F}^{0}} \rho(E, H=0) d E=\int_{-|\Delta \epsilon|}^{E_{F}} \rho(E, H) d E,
$$

where $E_{F}^{0}$ is the Fermi level at $H=0$. Substituting Eqs. (4) and (5) into the above equation, the Fermi energy can be determined from the following equation

$$
\int_{0}^{E_{F}^{0}} 2 \rho_{0}(E) d E=\left(\int_{0}^{E_{F}+|\Delta \epsilon|}+\int_{0}^{E_{F}-|\Delta \epsilon|}\right) \rho_{0}(E) d E .
$$

Now one can see that the total DOS at the new Fermi level $E_{F}$ is generally $H$ dependent

$$
\rho\left(E_{F}\right)=\rho_{0}\left(E_{F}-|\Delta \epsilon|\right)+\rho_{0}\left(E_{F}+|\Delta \epsilon|\right) .
$$

With the use of Eq. (1), one obtains the following MR

$$
M R(H) \equiv \frac{R(H)-R(0)}{R(0)}=\frac{\rho^{-1}\left(E_{F}\right)-2 \rho_{0}^{-1}\left(E_{F}^{0}\right)}{2 \rho_{0}^{-1}\left(E_{F}^{0}\right)},
$$

where $R(H)$ and $R(0)$ denote the resistance with and without external magnetic field, respectively. One can see that the MR depends on $\rho_{0}(E)$ and $E_{F}^{0}$, and $E_{F}^{0}$ is determined by $\rho_{0}(E)$ and carrier concentration which is given by the stoichiometric index $\delta$ in silver chalcogenides such as $\mathrm{Ag}_{2}+{ }_{\delta} \mathrm{Se}$. In other words, the MR is determined by the band strcuture, and we shall call it the "band effect."

Strictly speaking, the above arguments are valid only at low temperature. At high temperature the carriers will be activated, and the contribution to the conductivity will be extended to electrons with $\Delta E=\left|E-E_{F}\right| \leqslant k_{B} T, k_{B}$ the Boltzmann constant, $T$ the temperature. However, this mechanism should still exist although the effect is expected to be suppressed with the increase of temperature.

Let us discuss the conditions under which the band effect may be considerable. In order to obtain a substantial MR, the Zeeman energy should be large enough such that the Fermi energy can have a noticeable change in the magnetic field. Furthermore, the electron DOS should not be too smooth. As the Bohr magneton $\mu_{B}$ is very small $\left(\sim 10^{-6} \mathrm{meV} / \mathrm{Oe}\right)$, the Lande factor $g$ of the material should be large. The Fermi level $E_{F}^{0}$ at zero field should be close to the bottom of electron band. This requires carrier density to be low. For a silver chalcogenide, such as $\mathrm{Ag}_{2}+\delta \mathrm{Se}$, this means that the stoichiometric index $\delta$ should be small. For a conventional metal or a semiconductor, the Lande factor $g \sim 1$, and the band effect in such a material should be very small and undetectable. However, some semimetals and lightly doped narrow-gap semiconductors have a large Lande factor, and the band effect in these materials may be considerable. For example, the effective mass of silver chalcogenides $m$ is of the order of $10^{-2} m_{0}$ ( $m_{0}$ the mass of a free electron $)^{7}$ and the Lande factor $g$ is as large as $10^{2}$. In a magnetic field $H \sim 10^{4}$ Oe which is the same order of magnitude in Xu et al.'s experiment, the Zeeman energy is $\Delta \epsilon \sim \mathrm{meV}$, which can be comparable with the Fermi energy at very small $\delta$. Thus the band effect might be a possible mechanism for the large MR in those materials. In this case, the contribution to MR will finally reach a saturation at a high magnetic field $H_{c}$ when the Zeeman splitting converts all the electrons in the spindown part into the spin-up part. For even higher $H$, all the electrons remain in spin-up part, and it is clear that $\rho\left(E_{F}, H\right)$ does not change anymore [see Fig. 1(c)].

To illustrate the physical picture mentioned above, we carry out two model calculations. In the first model calcula- 


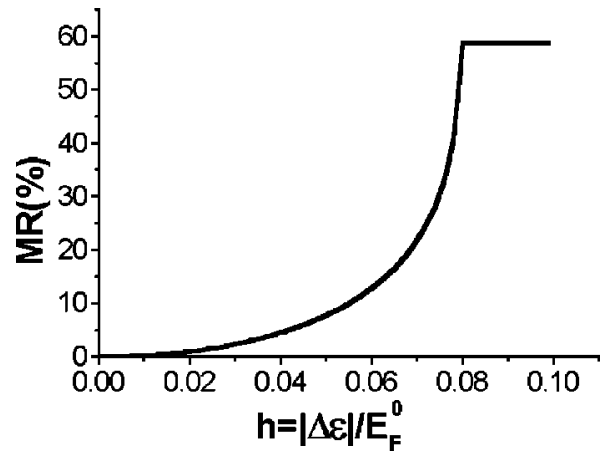

FIG. 2. The MR induced by Zeeman splitting for a conventional band edge $\rho_{0}(E)=E^{1 / 2}$.

tion, we assume a band with $\rho_{0}(E)=C E^{1 / 2}$. For convenience, we use the ratio of Zeeman energy and original Fermi energy $h \equiv|\Delta \epsilon| / E_{F}^{0}$ as the independent variable for the calculation of MR. We numerically solve Eq. (7) to obtain the $H$-dependent Fermi level $E_{F}$, and then calculate the MR with the use of Eq. (9). In the calculation, we neglect the coefficiency $C$ and just take $\rho_{0}(E)=E^{1 / 2}$. In fact, the coefficiency $C$ has no influence on the MR because it is just a constant, independent of $E$ and $H$. The result is plotted in Fig. 2. The MR is positive. It increases slowly when $h$ $<0.06$ while gets a sudden increase from $h \sim 0.06$ to $h$ $\sim 0.08$. Finally it reaches a saturation value of about $60 \%$ when $h \sim 0.08$. As the width of a conventional band is $\sim \mathrm{eV}$, for the case of silver chalcogenide with a small stoichiometric index $\delta \sim 0.01$ we may estimate that $E_{F}^{0}$ $\sim 0.01 \mathrm{eV}$. Then, when MR reaches $\sim 60 \%$, the Zeeman energy is $\sim 0.08 E_{F}^{0} \sim \mathrm{meV}$. As discussed before, the magnetic field in $\mathrm{Xu}$ et. al.'s experiment can induce a Zeeman energy of the same order of magnitude. Thus the band effect alone can considerably contribute to the MR in that experiment.

The second model calculation is on a band with $\rho_{0}(E)$ $=E^{3 / 2}$. The result is shown in Fig. 3. The MR is also positive, monotonically increasing and saturated at a value of about $30 \%$ when $h \sim 0.06$. However, unlike the first one, the increase of MR is steady in the whole region. Furthermore, it

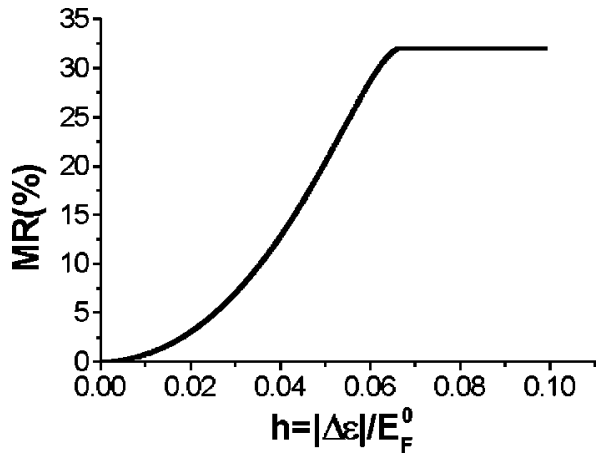

FIG. 3. The MR induced by Zeeman splitting for a band edge with $\rho_{0}(E)=E^{3 / 2}$.

shows a $h^{2}$-like behavior in low $h$ while changes into a linearlike one in relatively high $h$, which is similar to the observed MR behavior in silver chalcogenides. This shows that the behavior of MR is sensitive to the change of $\rho_{0}(E)$.

As another possibility, the band effect may also be considerable for a narrow band whose width is comparable to the Zeeman energy in a high magnetic field. We may consider the deep impurity band in some lightly doped semiconductors as an example. In this case the MR can reach very high value or even be divergent in high $H$ limit. For example, suppose that at zero field the narrow band is nearly halffilled. As discussed before, in a high magnetic field all electrons will be in the spin-up part, then the spin-up part will be nearly filled while the spin-down part is empty, leading to a nearly insulating behavior and a divergent MR.

In summary, we show that the change of the DOS at the Fermi energy due to the coupling of the electron spin and the external magnetic field $H$ can lead to a considerable MR when the original Fermi level is near the edge of the band and the Lande factor $g$ of electrons is large, which may be a possible mechanism for the large MR in nonmagnetic materials with a large Lande factor $g$ such as silver chalcogenides.

We would like to thank Dr. Y. Q. Wang for stimulating discussions. This work was supported by UGC, Hong Kong, through RGC/DAG and RGC/CERG grants.
${ }^{1}$ M.N. Baibich, J.M. Broto, A. Fert, F. Nguyen van Dau, F. Petroff, P. Etienne, G. Creuzet, A. Friedrich, and J. Chazelas, Phys. Rev. Lett. 61, 2472 (1988); J.Q. Xiao, J.S. Jiang, C.L. Chien, ibid. 68, 3749 (1992); P. Xiong, G. Xiao, J.Q. Wang, J.Q. Xiao, J.S. Jiang, and C.L. Chien, ibid. 69, 3220 (1992).

${ }^{2}$ A.E. Berkowitz, J.R. Mitchell, M.J. Carey, A.P. Young, S. Zhang, F.E. Spada, F.T. Parker, A. Hutten, and G. Thomas, Phys. Rev. Lett. 68, 3745 (1992).

${ }^{3}$ J.Q. Xiao, J.S. Jiang, and C.L. Chien, Phys. Rev. Lett. 68, 3749 (1992).

${ }^{4}$ A.J. Millis, B.I. Shraiman, and R. Mueller, Phys. Rev. Lett. 77, 175 (1996); Y. Tokura, J. Appl. Phys. 79, 5288 (1996).
${ }^{5}$ Kai Liu and C.L. Chien, Phys. Rev. B 58, 14681 (1998).

${ }^{6}$ Tineke Thio, S.A. Solin, J.W. Bennett, and D.R. Hines, Phys. Rev. B 57, 12239 (1998)

${ }^{7}$ R. Xu, A. Husmann, T.F. Rosenbaum, M.-L. Saboungi, J.E. Enderbya, and P.B. Littlewood, Nature (London) 390, 57 (1997).

${ }^{8}$ X.R. Wang, S.C. Ma, and X.C. Xie, Europhys. Lett. 45, 368 (1999).

${ }^{9}$ Z. Ogorelec, A. Hamzic, M. Basletic, Europhys. Lett. 46, 56 (1999).

${ }^{10}$ Y. Q. Wang and X. X. Zhang (private communication). 